\title{
Low power consumption quartz-enhanced photoacoustic gas sensor employing a quantum cascade laser in pulsed operation
}

\author{
Angelo Sampaolo $^{\mathrm{a}, \mathrm{b}}$, Pietro Patimisco ${ }^{\mathrm{a}, \mathrm{b}}$, Aleksander Gluszek ${ }^{\mathrm{b}, \mathrm{c}}$, Arkadiusz Hudzikowski ${ }^{\mathrm{b}, \mathrm{c}}$, Marilena \\ Giglio $^{\mathrm{a}, \mathrm{b}}$, Huadan Zheng ${ }^{\mathrm{b}}$, Frank K. Tittel ${ }^{\mathrm{b}}$ and Vincenzo Spagnolo ${ }^{\mathrm{a}}$ \\ ${ }^{a}$ Dipartimento Interateneo di Fisica, University and Politecnico of Bari, CNR-IFN UOS BARI, Via \\ Amendola 173, Bari, Italy; \\ ${ }^{b}$ Department of Electrical and Computer Engineering, Rice University, 6100 Main Street, Houston, \\ TX 77005, USA; \\ cElectronics Department, Wroclaw University of Technology, 50-370 Wroclaw, Poland
}

\begin{abstract}
We report here an analysis of the performance of a quartz-enhanced photoacoustic (QEPAS) system operating in a pulsed mode by employing a quantum cascade laser (QCL). The QEPAS system is based on a quartz tuning fork (QTF) having fundamental resonance frequency of $4.2 \mathrm{kHz}$ and a first overtone resonance of $25.4 \mathrm{KHz}$. Water vapor was used as a target gas by selecting its absorption line falling at $1296.5 \mathrm{~cm}^{-1}$ with a line strength of $1.69 \cdot 10^{-22} \mathrm{~cm} / \mathrm{molecule}$. The QEPAS signal was investigated, while varying the QCL duty-cycle from continuous wave operation, down to 5\%, which corresponds to a laser power consumption of $0.17 \mathrm{~mW}$ and a pulse-width of $4 \mu \mathrm{s}$.
\end{abstract}

Keywords: quartz tuning fork, photoacoustic spectroscopy, quantum cascade laser, gas sensing

\section{INTRODUCTION}

Gas sensing techniques based on laser absorption spectroscopy are excellent candidates for real-world applications requiring fast and in-situ measurements. When operating at atmospheric pressure, trace gas sensors based on optical techniques present two main sources of power consumption: a laser source that typically requires an air- or water-cooled system for temperature stabilization and a cooled infrared photodetector. Quartz-enhanced photo-acoustic spectroscopy (QEPAS) is the only laser-based technique that does not require an optical detector, since the quartz tuning fork (QTF) itself acts as an uncooled, wavelength insensitive detector with negligible power consumption [1,2]. Therefore, QEPAS is an ideal technique for the realization of trace gas sensors with low-power consumption. The power consumption of a QEPAS gas sensor system could be reduced even further if operating with pulsed laser sources, avoiding in this way the use of their cooling systems.

The possibility to employ pulsed laser sources for QEPAS can also be advantageous for the application of this sensing technique in the THz range, which represents the most promising spectral range in terms of sensitivity and selectivity [1, 3-6]. Up to now the only type of laser sources employed for THz QEPAS sensing are continuous-wave quantum cascade lasers (QCLs) [3-6]. However, although more than a decade of R\&D effort, THz QCLs still operate only at cryogenic temperature [7, 8]. The highest temperature operation reported to-date for a THz QCL is $199.5 \mathrm{~K}$ in a pulsed mode [9] and this temperature value decreases to $129 \mathrm{~K}$ in continuous-wave operation [10]. The main reason for THz QCLs limited thermal properties are the large threshold currents and voltages required, leading to strong local heating effects in the device active regions. Above threshold for continuous-wave (CW) and high duty-cycle operation, the device active region temperature becomes much higher than that of the heat sink $[11,12]$ and the consequent reduction of gain represents the major impediment for THz QCL room-temperature (RT) operation [8]. An alternative approach to achieve $\mathrm{RT}$ operation in the $\mathrm{THz}$ range consists in in high-power two-color mid-IR QC-lasers for intracavity $\mathrm{THz}$ difference frequency [13]. These sources are monolithic and operate at RT, and wide THz tunability can be obtained, however the highest output power reported to date is $1.9 \mathrm{~mW}$ in a pulsed mode and $14 \mu \mathrm{W}$ in a $\mathrm{CW}$ mode [14].

In contrast to continuous-wave QCLs, the line width of lasers operated in a pulsed mode is broadened due to thermal chirping. However, for applications, which do not require ultra-high selectivity, e.g. concentration measurements with

Quantum Sensing and Nano Electronics and Photonics XIV, edited by Manijeh Razeghi,

Proc. of SPIE Vol. 10111, 101110C · C 2017 SPIE · CCC code: 0277-786X/17/\$18 doi: $10.1117 / 12.2252326$ 
strongly pressure-broadened spectroscopic features or measurements at low pressure using well-isolated absorption lines, a pulsed QCL can be an adequate radiation source,

The landscape offered by state-of-art of THz QCLs suggests an interesting perspective. Both continuous wave and pulsed operations require low working temperatures in order to achieve high power or peak power for pulsed operations. However, in pulsed operation $\mathrm{THz}$ lasers provide output powers more than two orders of magnitude higher with respect to intracavity $\mathrm{THz}$ difference frequency QCL sources operating at room temperature [15]. The QEPAS technique has already proven to be effective also under pulsed operations [16-18]. The investigation reported in this work is focused on the characterization of the QEPAS response of a resonator in terms of signal amplitudes, signal shape and optical noise levels, when the duty cycle of a pulsed QCL is varied from the CW regime down to few $\mu$ s pulse widths. This investigation allowed us to analyze different sources of noise as well as the QEPAS signal shape and intensity, providing useful guidelines for the design of low power consumption sensors based on a pulsed modulation approach. The final goal of the study is the implementation of the pulsed modulation approach for THz QEPAS sensing.

\section{PULSED QUANTUM CASCADE LASER}

Quantum cascade lasers (QCLs) are characterized by the ultrafast carrier lifetimes (on the order of picoseconds), which means that an ultra-wide range of modulation frequencies up to $\mathrm{GHz}$ should be possible [19]. The thermal effects in a traditional mid-IR QCL active region are described by the heat diffusion equation in two dimensions [20]. For frequencies in the order of few tens of $\mathrm{kHz}$ and pulsed duration below $1 \mu$ s the active region temperature is nearly independent of the pulse frequency. This is due to the relatively long time between pulses, which allows the active region to dissipate the heat accumulated during the pulse and recover the heat sink temperature [21]. Since in QEPAS the current modulation frequencies are set by the QTF flexural mode resonances (typically $<40 \mathrm{kHz}$ ), the only parameter to work with, to limit the QCL temperature increases, is the pulse duration. For pulse-widths in the order of tens of nanoseconds, negligible active region heating occurs. Above this value, the active region temperature can increase of tens of ${ }^{\circ} \mathrm{C}$. For example in [20] it was shown that for a pulse-width of 100-ns pulse and a modulation frequency of 1 $\mathrm{MHz}$ (duty cycle of $10 \%$ ), starting from a heat sink temperature of $270 \mathrm{~K}$, resulted in a maximum active region temperature of $370 \mathrm{~K}$. The emission wavelength of a DFB laser is given by the Bragg grating period $\Lambda$ and by the temperature-dependent effective refractive index of the waveguide mode $n_{e f f}(T)$ :

$$
\lambda(T)=2 \Lambda n_{\text {eff }}(T)
$$

When DFB QCLs operates in pulsed mode, a blue-shift of the emission wavelength with respect to CW mode is expected, due to the lower dissipated electrical power, which corresponds to lower active region temperatures. The wavelength shift during a single pulse of $1 \mu \mathrm{s}$ can be as high as $1 \mathrm{~cm}^{-1}[22,23]$. Furthermore, it was demonstrated that operating in pulsed mode, a broadening of the emission occurs because of current-induced self-heating of the QCL during each pulse. With QCLs in continuous-wave operation, it is possible to obtain linewidths of $<1 \mathrm{MHz}$, limited by the spectral noise induced by the current driver. In pulsed operation, the smallest linewidth (obtained with $<10$ ns-long pulsed) lies in the few hundreds of MHz range.

\section{EXPERIMENTAL SETUP}

The schematic of the QEPAS setup is shown in Fig. 1. The laser source employed in this work was a mid-IR DFB QCL emitting at $7.8 \mu \mathrm{m}$, driven by a custom-made current pulse generator with a pulse rise-time of $50 \mathrm{~ns}$. The collimated laser beam was focused between the prongs of the quartz tuning fork (QTF) by means of a lens with a focal length of $50 \mathrm{~mm}$. The QTF is located in an enclosure equipped with two windows. The QTF has a prong length of $19 \mathrm{~mm}$ and width of 1.4 $\mathrm{mm}$ was employed as the acousto-electric transducer in the QEPAS system [24]. The QTF thickness is $0.8 \mathrm{~mm}$ and the space between the prongs is $1.0 \mathrm{~mm}$. The fundamental and $1^{\text {st }}$ overtone modes resonance frequencies of the investigated custom QTF fall at $\mathrm{f}_{0} \sim 4.25 \mathrm{kHz}$ and $\mathrm{f}_{1} \sim 25.4 \mathrm{kHz}$, respectively [24, 25]. The light exiting from the housing is collected by a lens and focused on a mid-infrared detector or a pyrocamera for alignment.

For our investigation, water vapor was selected as gas target. The $\mathrm{H}_{2} \mathrm{O}$ concentration was fixed at $1.7 \%$ by using a Nafion humidifier. A hygrometer was connected to the upstream side of the gas cell in order to monitor the water vapor content. The QEPAS sensor was operated at atmospheric pressure. The selected absorption line falls at $1296.7 \mathrm{~cm}^{-1}$ with

line strength of $1.69 \cdot 10^{-22} \mathrm{~cm} /$ molecule. At a $20^{\circ} \mathrm{C}$ heat sink temperature and $\mathrm{CW}$ operation, a QCL current of $\mathrm{I}=263 \mathrm{~mA}$ allows the laser emission to be resonant with the selected $\mathrm{H}_{2} \mathrm{O}$ absorption line. 


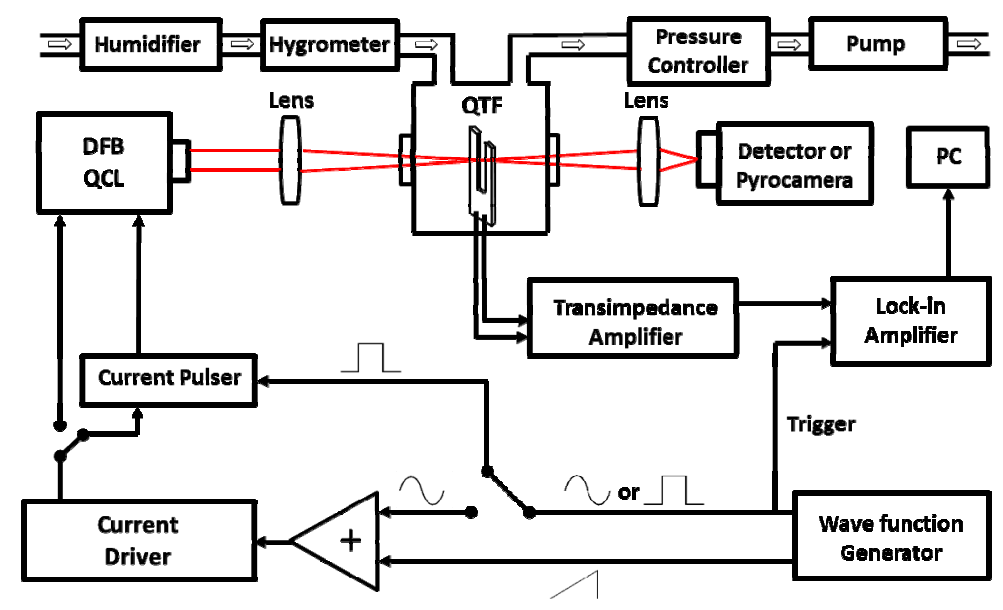

Figure 1. Schematic of the QEPAS gas sensor system using a QCL as the excitation source. PC: Personal Computer

QEPAS CW measurements were performed in a wavelength modulation and $2 f$-detection approach. A sinusoidal waveform provided by a function generator (Tektronix model AFG3102) at half of the QTF resonance frequency $f / 2$ is applied to the current driver, while the QTF response is demodulated at $f$ by using a lock-in amplifier (Stanford Research Model SR830). For pulsed QEPAS, a square-wave signal was applied to the custom current pulser to turn the laser on and off at a frequency $\mathrm{f} / 2$ while the QTF response is demodulated at $\mathrm{f}$ to provide the QEPAS signal. The frequency of the square-wave signal applied to the current pulser must be coincident to one of the QTF resonances. We decided to operate the QTF at the first overtone flexural mode $\left(f_{l}=25.4 \mathrm{kHz}\right)$, since it provides the highest QEPAS performance [26]. The laser beam must be focused at one of two antinodes points of the vibration mode profile, where the maximum vibration amplitude is generated [25-28]. Preliminary QEPAS measurements were performed by scanning the laser focusing position along the QTF symmetry axis in steps down to $250 \mu \mathrm{m}$ and measuring the associated QEPAS signal. The position that maximizes the QEPAS signal occurs at $10.5 \mathrm{~mm}$ from the top of the prongs, corresponding to the lower antinode point of the first overtone mode [26]. A slow voltage ramp allows scanning of the laser wavelength through the selected water absorption line. All instruments were controlled by LabView-based software.

\section{SPECTRAL CHARACTERISTICS OF THE PULSED QCL}

The temperature-induced wavelength shift of the DFB QCL in pulsed operation was studied by fixing the heat sink temperature at $20^{\circ} \mathrm{C}$, the repetition rate to $12.7 \mathrm{kHz}\left(f_{I} / 2\right)$ and the injected current $I=263 \mathrm{~mA}$, while varying the duty cycle from $\sim 50 \%$ (pulse width of $40 \mu \mathrm{s}$ ) to $\sim 5 \%$ (pulse-width of $4 \mu \mathrm{s}$ ). The measured QCL spectra, acquired by using an FTIR spectrometer are shown in Fig. 2a).
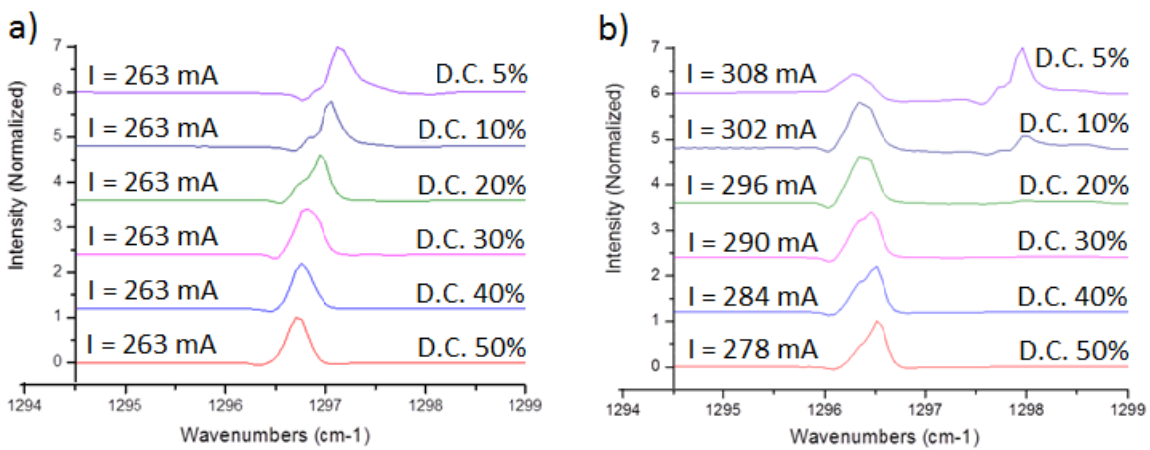

Figure 2. a) QCL spectral characteristics at $\mathrm{T}=20^{\circ} \mathrm{C}, \mathrm{I}=263 \mathrm{~mA}$ and repetition rate to $12.7 \mathrm{kHz}$, for different duty cycles (D.C.); b) QCL spectral characteristics at $\mathrm{T}=20^{\circ} \mathrm{C}$ and different currents for each duty cycle. These currents were chosen to keep the laser emission wavelength centred at $\sim 1296.5 \mathrm{~cm}^{-1}$. 
We observed a blue-shift of the emission wavelength, when moving from $50 \%$ down to $5 \%$ duty cycle. Therefore, in order to keep the emission wavelength centered at the selected water absorption line an adjustment of the injection currents was needed. For the different duty cycles, the injected currents needed to fix the laser emission at $1296.5 \mathrm{~cm}^{-1}$ and the related dissipated powers are listed in Table 1, while the spectral characteristics of the emission are shown in Fig. $2 b)$.

Table 1: Duty cycles with relative currents and power consumption when the QCL central emission wavelength falls at $1296.5 \mathrm{~cm}^{-1}$.

\begin{tabular}{|c|c|c|}
\hline Duty Cycle & Current $(\mathbf{m A})$ & Power consumptions $(\mathbf{W})$ \\
\hline $\mathbf{C W}$ & 263 & 2.76 \\
\hline $\mathbf{5 0} \%$ & 278 & 1.50 \\
\hline $\mathbf{4 0 \%}$ & 284 & 1.24 \\
\hline $\mathbf{3 0 \%}$ & 290 & 0.96 \\
\hline $\mathbf{2 0 \%}$ & 296 & 0.66 \\
\hline $\mathbf{1 0 \%}$ & 302 & 0.34 \\
\hline $\mathbf{5 \%}$ & 308 & 0.17 \\
\hline
\end{tabular}

When the duty-cycle decreases, the injected current increases in order to lock the laser emission wavelength to 1296.5 $\mathrm{cm}^{-1}$. At $50 \%$ duty cycle, we observed a linewidth as broad as $0.3 \mathrm{~cm}^{-1}$ of the emission spectra. This broadening increased when the pulse width is reduced. For duty-cycles lower than $10 \%$ the QCL emission becomes multimodal. As a result, QEPAS spectra profiles and background noise level will be affected.

The background noise level is also affected by the spatial quality of the beam profile focused between the QTF prongs. In fact, the portion of laser light hitting the QTF generates an undesirable non-zero background [1]. Thus, it is important to employ a laser beam with quality as high as possible to avoid hitting the QTF prongs. We measured the beam profile at different duty cycles using a pyrocamera positioned $3 \mathrm{~cm}$ far from the QCL. Representative measured beam profiles are shown in Fig. 3.

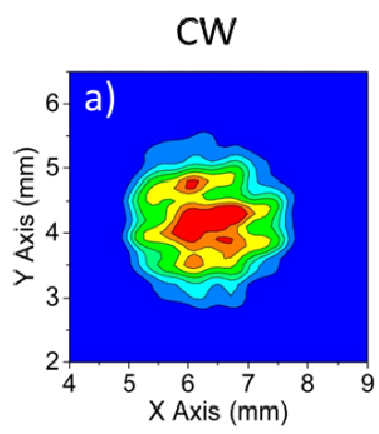

\section{Duty Cycle 50\%}

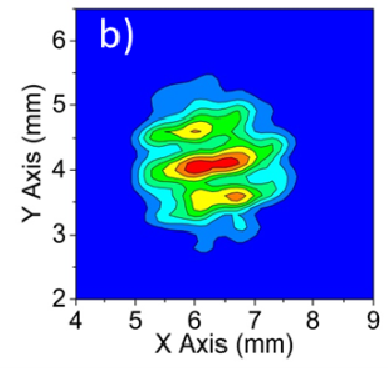

Duty Cycle $30 \%$

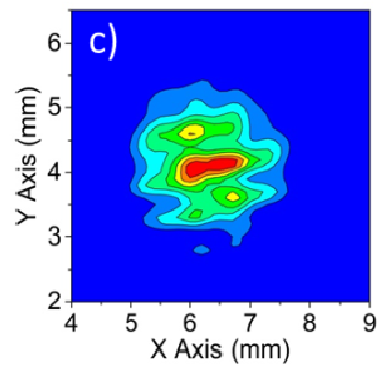

\section{Duty Cycle 5\%}

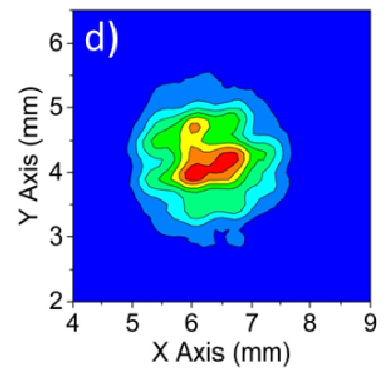

Figure 3. (a-d) Beam profiles of the QCL emitting in CW (a), at 50\% duty cycle (b), at $30 \%$ duty cycle (c), and at $5 \%$ duty cycle (d), recorded by positioning the infrared pyrocamera $3 \mathrm{~cm}$ away from the QCL exit.

We observed that the laser beam profile slightly changes with the duty cycle. The laser focusing condition between the 
QTF prongs was optimized under CW operation and $99.5 \%$ of the laser light passed between the prongs. With the laser operated in pulsed mode the portion of the transmitted light is reduced due to a change in power distribution profile of the QCL beam and dropped down to $98.8 \%$ at $5 \%$ duty cycle. As a consequence the QTF background noise level will be influenced by the selected duty cycle.

\section{PULSED QEPAS}

In Fig. 4a), the simulated absorption spectra for a gas mixture at of standard air and $1.7 \%$ of $\mathrm{H}_{2} \mathrm{O}$ at atmospheric pressure in the range $1296 \mathrm{~cm}^{-1}-1297.1 \mathrm{~cm}^{-1}$, using HITRAN database [29] is shown. Two peaks at 1296.5 $\mathrm{cm}^{-1}$ and $1296.7 \mathrm{~cm}^{-1}$ related to the water vapor are visible. .

In Fig. 4b), we reported the QEPAS spectra measured with the laser operated in CW mode with $\mathrm{I}=263 \mathrm{~mA}$ at $\mathrm{T}=20$ ${ }^{\circ} \mathrm{C}$. A ramp of $250 \mathrm{mV}$ and a sinusoidal dither at $\mathrm{f}_{1} / 2$ with a peak-to-peak amplitude of $100 \mathrm{mV}$ was simultaneously applied to the laser current. The focused laser power between the prongs of the QTF was $110 \mathrm{~mW}$.
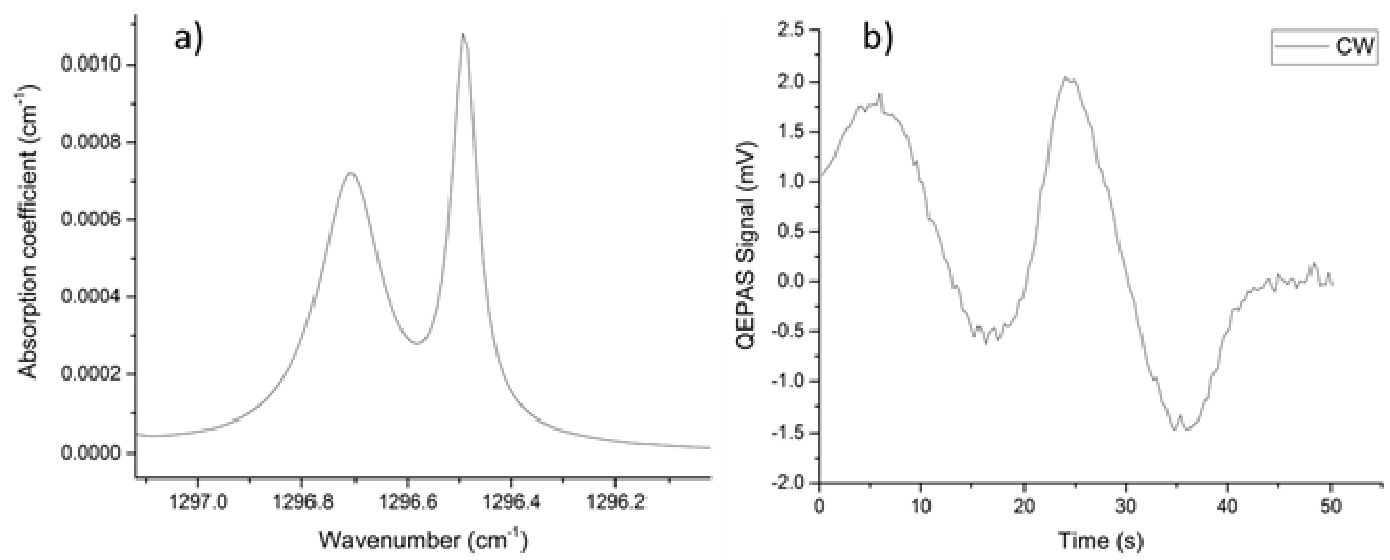

Figure 4. a) HITRAN simulation of the absorption spectra calculated for a gas mixture of $1.7 \%$ of $\mathrm{H}_{2} \mathrm{O}$ in standard air at atmospheric pressure. The peaks at $1296.5 \mathrm{~cm}^{-1}$ and $1296.7 \mathrm{~cm}^{-1}$ are due to water vapor. b) Wavelength modulation QEPAS spectrum measured for the same gas mixture and pressure, with the QCL source operating in a CW condition and emission centred at $1296.5 \mathrm{~cm}^{-1}$.

The QEPAS CW spectral scan in Fig. 4b) resembles a $2^{\text {nd }}$ derivative-like shape of the absorption line at $1296.5 \mathrm{~cm}^{-1}$. The asymmetry between the two minima is due to the partial overlap with the absorption line falling at $1296.7 \mathrm{~cm}^{-1}$, which makes the first minima higher than the second and displaying a first absorption peak (at $5 \mathrm{~s}$ ) in place of the background level visible at the end of the ramp.

The full-width-half-maximum (FWHM) of the absorption line at $1296.5 \mathrm{~cm}^{-1}$ is $0.056 \mathrm{~cm}^{-1}$, several times lower than the laser linewidth $\Delta v$, when it operates in a pulsed mode. The Taylor series expansion of the absorption coefficient, typically employed to predict the line shape and the background level of QEPAS spectra, cannot be applied since it is valid only if $\Delta v<<$ FWHM. This means that, even if the wavelength modulation and $2 f$-detection technique is employed, the QEPAS pulsed spectra could not exhibit the second-derivative of a Lorentzian line shape and cannot be background-free.

There is another aspect that must be considered related to the operation with square-wave current pulses. A pulsed excitation in fact, introduces a bunch of unwanted multiples of the modulation frequency $f_{1} / 2$ as shown in Fig. 5. The Fourier transform of a burst of 200 pulses for a duty cycle of 5\% and 50\% compared as extreme cases of a set of measurements. Additional duty cycles of $40 \%, 30 \%, 20 \%, 10 \%$ were also analyzed. For a duty cycle of $50 \%$, only a slight portion of the optical power is distributed to the harmonics of $f_{1} / 2$. This portion increases as the duty cycle decreases and becomes comparable to the power modulated at $\mathrm{f}_{1} / 2$ itself, as shown in Fig. 5 for a $5 \%$ duty cycle. This means that the QCL power is dispersed over a dense spectrum of frequency components that does not contribute to the QEPAS signal at the resonance frequency. This is expected to further reduce the QEPAS peak signal as the duty cycle decreases. 


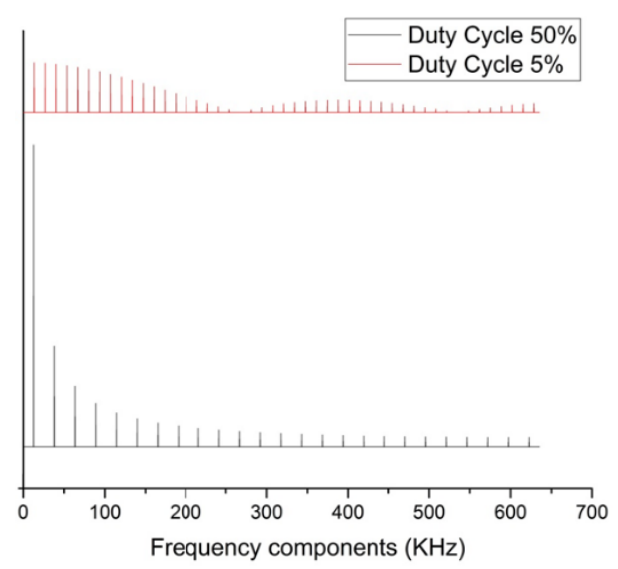

Figure 5. Frequency components introduced by pulses at $12.7 \mathrm{KHz}$ and $4 \mu$ s wide (5\% Duty Cycle, red line) and pulses at the same frequency and $39 \mu$ s wide (50\% Duty Cycle, black line).

In Figure 6 are shown the 2f-QEPAS spectral scans of the selected absorption line obtained for different duty cycles.
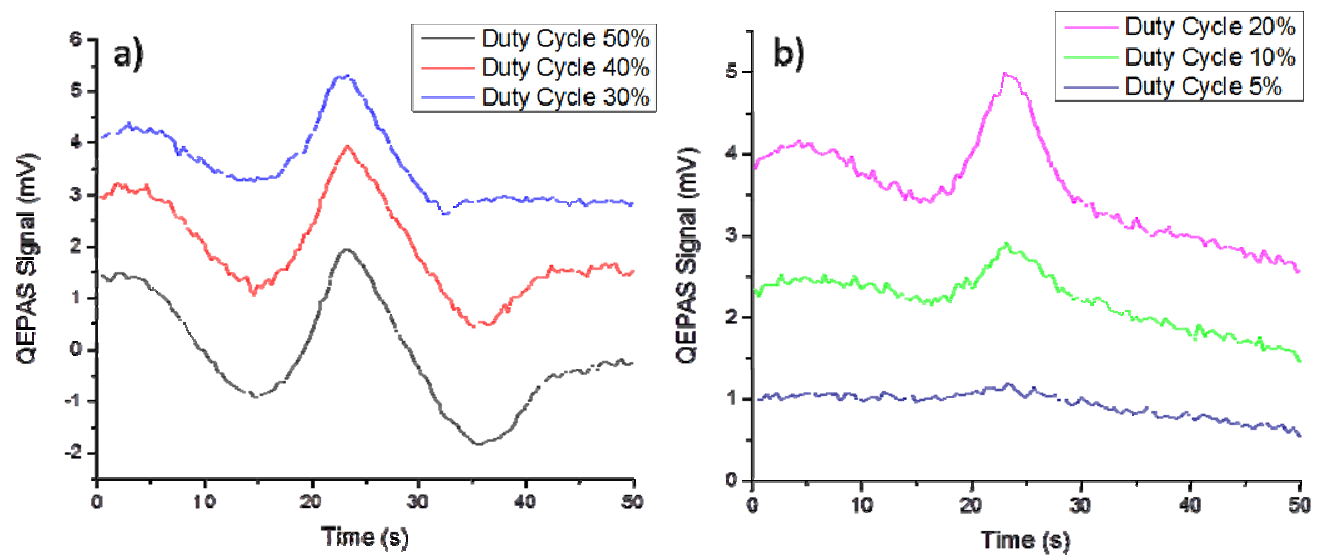

Figure 6. Wavelength modulation QEPAS spectra measured a gas mixture of $1.7 \%$ of $\mathrm{H}_{2} \mathrm{O}$ in standard air at atmospheric pressure with the QCL source operating in pulsed mode with a duty cycle from $50 \%$ to $5 \%$.

The 2f-QEPAS spectrum measured at a duty cycle of $50 \%$ is similar in intensity and shape to the one recorded in CW (see Fig. 4b)). Decreasing the duty cycle to $20 \%$, an overall rise of the background noise level was observed. The QEPAS peak signal scales with the QCL optical power for duty cycles down to $20 \%$, while for lower duty cycles the peak signal decreases more rapidly. This is due to the reduced portion of optical power modulated at $\mathrm{f}_{1} / 2$ and the QCL multimode behavior (see Fig. 2b). Below $20 \%$ duty cycle, part of the laser power (more than $50 \%$ at $5 \%$ duty cycle) is channeled in the laser mode emitting at $\sim 1298 \mathrm{~cm}^{-1}$, far from the resonance of the $\mathrm{H}_{2} \mathrm{O}$ absorption lines and hence not contributing to the QEPAS signal. The employed QCL was not designed to be operated in a pulsed mode and in fact, we did not observe an enhancement of the ratio between the QEPAS peak signal and the average QCL optical power when reducing the duty cycle. For QCLs properly designed for pulsed operations, as typical in the THz range, an enhancement in peak power at short pulses is expected. The possibility to operate with pulsed THz QCL sources will also widen their operating temperature range.

\section{CONCLUSIONS}

In this work, we investigated the performance of a QEPAS sensor system employing a custom QTF with a prong spacing of $700 \mu \mathrm{m}$ and a mid-IR DFB QCL source operating in both CW and pulsed conditions. We examined the influence of the duty cycle operation on the QCL spectral properties and QEPAS signal profile as well as analyzed the main sources 
of instrumental background noise. These analyses can be used as a guideline for the realization of pulsed QEPAS sensors operating with QCLs with particular interest in the THz spectral range.

\section{ACKNOWLEDGEMENTS}

Frank Tittel acknowledges support by the Welch Foundation (Grant R4925S). The authors from Dipartimento Interateneo di Fisica di Bari acknowledge financial support from two Italian research projects: PON02 00675 and PON02 00576. The authors from Shanxi University acknowledges the support by National Natural Science Foundation of China 61622503, the Education Innovation Project of Shanxi Province (2016BY028).

\section{REFERENCES}

[1] Patimisco, P., Scamarcio, G., Tittel, F.K., Spagnolo, V., "Quartz-Enhanced Photoacoustic Spectroscopy: A Review," Sensors 14, 6165-6206 (2014).

[2] Kosterev, A.A., Tittel, F.K., Serebryakov, D., Malinovsky, A., and Morozov, A., "Applications of quartz tuning fork in spectroscopic gas sensing," Rev. Sci. Instrum. 76, 043105:1-043105:9 (2005).

[3] Borri, S., Patimisco, P., Sampaolo, A., Beere, H.E., Ritchie, D.A., Vitiello, M.S., Scamarcio, G., and Spagnolo, V., "Terahertz quartz enhanced photo-acoustic sensor," Appl. Phys. Lett.103, 021105 (2013).

[4] Patimisco, P., Borri, S., Sampaolo, A., Beere, H.E., Ritchie, D.A., Vitiello, M.S., Scamarcio, G., and Spagnolo, V., "A quartz enhanced photo-acoustic gas sensor based on a custom tuning fork and a terahertz quantum cascade laser," Analyst 1339, 2079-2087 (2014).

[5] Spagnolo, V., Patimisco, P., Pennetta, R., Sampaolo, A., Scamarcio, G., Vitiello, M.S., and Tittel, F.K., "THz Quartz-enhanced photoacoustic sensor for $\mathrm{H}_{2} \mathrm{~S}$ trace gas detection," Opt. Express 23, 7574-7582 (2015).

[6] Sampaolo, A., Patimisco, P., Giglio, M., Vitiello, M.S., Beere, H.E., Ritchie. D.A., Scamarcio, G., Tittel, F. K., and Spagnolo, V., "Improved Tuning Fork for Terahertz Quartz-Enhanced Photoacoustic Spectroscopy," Sensors, 16, 439 (2016).

[7] Vitiello, M.S., Scalari, G., Williams, B., De Natale, P., "Quantum cascade lasers: 20 years of challenges," Opt. Express 23, 5167-5182 (2015).

[8] Williams, B.S., "Terahertz quantum-cascade lasers," Nat. Photonics 5, 17-25 (2007).

[9] Fathololoumi, S., Dupont, E., Chan, C.W.I., Wasilewski, Z.R., Laframboise, S.R., Ban, D., Mátyás, A., Jirauschek, C., Hu, Q. and Liu, H.C., "Terahertz quantum cascade lasers operating up to $\sim 200 \mathrm{~K}$ with optimized oscillator strength and improved injection tunneling," Opt. Express 20, 3866-3876 (2012).

[10] Wienold, M., Röben, B., Schrottke, L., Sharma, R., Tahraoui, A., Biermann, K., and Grahn, H.T., "Hightemperature, continuous-wave operation of terahertz quantum-cascade lasers with metal-metal waveguides and third-order distributed feedback," Opt. Express 22, 3334-3348 (2014).

[11] Evans, A.C., Indjin, D., Ikonic, Z., Harrison, P., Vitiello, M.S., Spagnolo, V., Scamarcio, G., "Thermal Modeling of Terahertz Quantum-Cascade Lasers: Comparison of Optical Waveguides," IEEE J. Quant. Electron. 44, 680 (2008).

[12] Vitiello, M.S., Scamarcio G., and Spagnolo, V., "Temperature dependence of thermal conductivity and boundary resistance in THz quantum cascade lasers," IEEE J. Sel. Topics Quantum Electron. 14, 431-435 (2008).

[13] Belkin, M.A., Capasso, F., Xie, F., Belyanin, A., Fischer, M., Wittmann, A., and Faist, J., "Room temperature terahertz quantum cascade laser source based on intracavity difference-frequency generation," Appl. Phys. Lett. 92, 201101 (2008).

[14]Lu, Q., and Razeghi, M., "Recent Advances in Room Temperature, High-Power Terahertz Quantum Cascade Laser Sources Based on Difference-Frequency Generation," Photonics 3, 42 (2016).

[15]Li, L., Chen, L., Zhu, J., Freeman, J., Dean, P., Valavanis, A., Davies, A.G., and Linfield, E.H., "Terahertz quantum cascade lasers with $>1 \mathrm{~W}$ output powers," Electron. Lett. 50, 309-311 (2014).

[16] Kosterev, A.A., Buerki, P.R., Dong, L., Reed, M., Day, T., Tittel, F.K., "QEPAS detector for rapid spectral measurements," Appl. Phys. B 100, 173-190 (2010).

[17] Bauer, C., Willer, U., Lewicki, R., Pohlkötter, A., Kosterev, A.A., Kosynkin, D., Tittel, F.K., Schade., W., “A Mid-infrared QEPAS sensor device for TATP detection,” J. Phys. Conf. Ser. 157, 012002 (2009). 
[18]Lassen, M., Lamard, L., Feng, Y., Peremans, A., Petersen, J.C., "Off-axis QEPAS using a pulsed nanosecond Mid-Infrared Optical Parametric Oscillator," Opt. Lett. 41, 4118-4121 (2016).

[19] Capasso, F., Paiella, R., Martini, R., Colombelli, R., Gmachl, C., Myers, T.L., Taubman, M.S., Williams, R.M., Bethea, C.G., Unterrainer, K., Hwang, H.Y., Sivco, D.L., Cho, A.Y., Sergent, A.M., Liu, H.C., Whittaker, E.A., "Quantum-cascade lasers: Ultrahigh-speed operation, optical wireless communication, narrow linewidth, and far-infrared emission," IEEE J. Quant. Electron. 38, 511-532 (2002).

[20] Evans, C.A., Jovanovic, V.D., Indjin, D., Ikonic, Z., Harrison P., "Investigation of Thermal Effects in QuantumCascade Lasers," IEEE J. Quant. Electron. 42, 857-865 (2006).

[21] Vitiello, M.S., Scamarcio, G., Spagnolo, V., "Time-resolved measurement of the local lattice temperature in terahertz quantum cascade lasers," Appl. Phys. Lett. 92, 101116 (2008).

[22] Pflugl, C., Schrenk, W., Anders, S., Strasser, G. "Spectral dynamics of distributed feedback quantum cascade lasers," Semicond. Sci. Tech. 19, S336-S338 (2004).

[23] Faist, J. Gmachl, C., Capasso, F., Sirtori, C., Sivco, D.L., Baillargeon, J.N., Cho, A.Y., "Distributed feedback quantum cascade lasers," Appl. Phys. Lett. 70, 2670 (1997).

[24] Patimisco, P., Sampaolo, A., Dong, L., Giglio, M., Scamarcio, G., Tittel, F.K., and Spagnolo, V., “Analysis of the electro-elastic properties of custom quartz tuning forks for optoacoustic gas sensing," Sensor Actuat. BChem. 227, 539-546 (2016).

[25] Tittel, F.K., Sampaolo, A., Patimisco, P., Dong, L., Geras, A., Starecki, T., and Spagnolo, V., "Analysis of overtone flexural modes operation in quartz-enhanced photoacoustic spectroscopy," Opt. Express 24, A682A692 (2016).

[26] Patimisco, P., Sampaolo, A., Zheng, H., Dong, L., Tittel, F.K. and Spagnolo V., "Quartz enhanced photoacoustic spectrophones exploiting custom tuning forks: a review", Adv. Phys. X, submitted (2016).

[27] Sampaolo, A., Patimisco, P., Dong, L., Geras, A., Scamarcio, G., Starecki, T., Tittel, F. K., and Spagnolo, V., "Quartz-enhanced photoacoustic spectroscopy exploiting tuning fork overtone modes," Appl. Phys. Lett. 107, $231102(2015)$.

[28]Zheng, H., Dong, L., Sampaolo, A., Wu, H., Patimisco, P., Ma, W., Zhang, L., Yin, W., Xiao, L., Spagnolo, V., Jia, S., and Tittel, F. K., "Overtone resonance enhanced single-tube on-beam quartz enhanced photoacoustic spectrophone, “ Appl. Phys. Lett. 109, 111103 (2016).

[29] http://www.hitran.org/ 